\title{
Better sorry than safe: Making a Plan B reduces effectiveness of implementation intentions in healthy eating goals
}

Charlotte D.W. Vinkers, Marieke A. Adriaanse, Floor M. Kroese \& Denise T.D. de Ridder

To cite this article: Charlotte D.W. Vinkers, Marieke A. Adriaanse, Floor M. Kroese \& Denise T.D. de Ridder (2015) Better sorry than safe: Making a Plan B reduces effectiveness of implementation intentions in healthy eating goals, Psychology \& Health, 30:7, 821-838, DOI: $\underline{10.1080 / 08870446.2014 .997730}$

To link to this article: http://dx.doi.org/10.1080/08870446.2014.997730

Accepted author version posted online: 07 Jan 2015.

Published online: 28 Jan 2015.

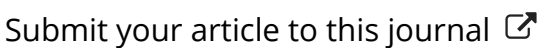

山 Article views: 249

Q View related articles

View Crossmark data $\asymp$ 


\title{
Better sorry than safe: Making a Plan B reduces effectiveness of implementation intentions in healthy eating goals
}

\author{
Charlotte D.W. Vinkers*, Marieke A. Adriaanse, Floor M. Kroese and \\ Denise T.D. de Ridder \\ Department of Clinical and Health Psychology, Utrecht University, Utrecht, The Netherlands \\ (Received 27 February 2014; accepted 5 December 2014)
}

\begin{abstract}
Objective: Implementation intentions (if-then plans) are helpful to health behaviour change. As these plans specify only one goal-directed behaviour for one specific situation, however, their effectiveness may be limited when a planned behaviour is impossible to execute in situ. The present research examines whether and how planning more than one goal-directed response for the same situation ('making a Plan B') affects successful self-regulation of eating behaviour.

Design and main outcome measures: In Study 1, participants formulated either one or two plans, after which a lexical decision task was administered to assess association strength between the if-part and the then-part(s). In Study 2 , the effect of making one, two or no plan(s) was assessed on actual eating behaviour, after which a Stroop task measured cognitive load as an additional explanatory mechanism.

Results: Study 1 revealed that making a Plan B disrupts the creation of strong if-then associations during plan formation. Study 2 showed that making a Plan B yields increased unhealthy food intake compared to making one or no plan, and induces greater cognitive load during plan enactment.

Conclusion: Making a Plan B interferes with essential cognitive processes during different stages of planning, leading to an increased likelihood of self-regulatory failure.
\end{abstract}

Keywords: implementation intentions; self-regulation; goal pursuit; planning; eating behaviour

Successful goal pursuit requires both flexibility and tenacity (Brandstädter \& Rothermund, 2004). Flexibility is necessary to adapt behaviour to the dynamic circumstances of goal pursuit, such as switching from fruitless to more fruitful courses of action (Wrosch, Scheier, Miller, Schulz, \& Carver, 2003). At the same time, goal pursuit requires tenacity to do as intended and to avoid falling prey to obstacles that interfere with goal pursuit (Shah, Friedman, \& Kruglanski, 2002). Implementation intentions, i.e. specific if-then plans (Gollwitzer, 1999), promote tenacity in goal pursuit, but their focus on one goal-directed behaviour (e.g. eating an apple) to be performed in one critical situation (e.g. watching TV) seems to defy flexibility. In this research, we aim to examine whether planned flexibility constitutes a viable solution to this problem.

*Corresponding author. Email: charlotte.vinkers@philips.com

Vinkers is a research scientist at Philips Research, Department of Behavior, Cognition, and Perception, in Eindhoven, The Netherlands. 
Specifically, we investigate whether and how planning more than one goal-directed response for a situation affects goal pursuit.

Implementation intentions are plans that specify when, where and how a goal will be pursued, for example, 'If it is Tuesday at 4 o'clock, then I will go jogging!' (Gollwitzer, 1999). By if-then planning, a mental link is created between the situation and the response, such that upon encountering the situation, the response will automatically be initiated (Bayer, Achtziger, Gollwitzer, \& Moskowitz, 2009). One type of implementation intentions, coping-oriented implementation intentions (CII), particularly help people successfully deal with obstacles that may derail goal pursuit (Gollwitzer, 1999). CII specify a situation that threatens goal adherence in the if-part and a coping response to deal with the goal threat in the then-part, e.g. 'If I am tired and feel like eating chocolate, then I will take an apple instead!'. In this article, we focus on such $\mathrm{CII}$ as they are especially suitable for health goals that are rife with goal-threatening situations, such as healthy eating goals - the topic of our research.

The effectiveness of implementation intentions for health behaviour change has been shown for various health behaviours including unhealthy eating (Adriaanse, Vinkers, de Ridder, Hox, \& de Wit, 2011). However, the defining feature of implementation intentions - the specification of one situation and one response - can be a double-edged sword. Although this one-on-one link ensures that goal-directed behaviour will be initiated without the need for deliberation, cognitive resources or conscious intent (Bayer et al., 2009), recent evidence demonstrates that it also discourages the use of goal-relevant behaviours that are not specified in the plan (Bayuk, Janiszewski, \& Leboeuf, 2010; Masicampo \& Baumeister, 2012). Specifically, when people have made an implementation intention, they are unlikely to perform unplanned behaviours, even if these unplanned behaviours facilitate goal pursuit. This raises the question whether implementation intentions' effects can be maximised by planning more than one response for a situation (henceforth referred to as 'making a Plan B').

Making a Plan B entails that people, in addition to making one implementation intention (e.g. 'If I feel like eating chocolate, then I will eat an apple'), formulate another implementation intention with the same situation specified in the if-part but a different response in the then-part (e.g. 'If I feel like eating chocolate, then I will distract myself'). Intuitively, making a Plan B seems a viable way to promote the much needed flexibility in goal striving that one implementation intention may hamper (Hagger \& Luszczynska, 2014). Although the potential benefits of planning multiple responses for one situation have been suggested by a review (Webb, 2006) and one unpublished study (Wieber, Odenthal, \& Gollwitzer, 2009; as cited in Gollwitzer, Wieber, Myers, \& McCrea, 2010), it is more likely that making a plan B harms rather than boosts goal attainment, as will be argued below.

There are several reasons why making a Plan B would be counterproductive. First, the formation of multiple responses linked to one situation may prevent the creation of strong mental associations between the situation and each response. In other words, making a Plan B may 'dilute' the associative strength between the situation and each planned response (Webb, 2006). Such weakened associations may decrease the likelihood that any of the responses will be executed, as strong mental links are a prerequisite for implementation intentions' effects (Gollwitzer, 1999). In line with this suggestion, it has been demonstrated that formulating multiple implementation intentions led to weakened associative links between situations and responses, thereby 
compromising successful goal attainment (Verhoeven, Adriaanse, De Ridder, De Vet, \& Fennis, 2013). Second, making a Plan B may negatively affect motivation to execute the plan(s) (Gollwitzer, 2006). Indeed, Dalton and Spiller (2012) showed that making multiple plans compromises the motivation necessary for successful goal pursuit.

The above two studies (Dalton \& Spiller, 2012; Verhoeven et al., 2013) provide indirect evidence that making a Plan B is more likely to be harmful rather than helpful to goal pursuit, as they showed that multiple implementation intentions are less beneficial to goal pursuit than one. However, these studies show divergent evidence as to why multiple plans are disadvantageous to goal pursuit: the negative effects of making more than one plan can be cognitive (Verhoeven et al., 2013) or motivational (Dalton $\&$ Spiller, 2012). A third mechanism that has been unexplored to date is that making a Plan B may interfere with processes during plan enactment rather than during plan formation. Gollwitzer (2006) suggests that multiple responses linked to one situation may create 'response competition'. This means that upon encountering the situation, the two competing responses interfere with the automatic enactment of planned behaviour (s), and hence, hinder goal attainment. Particularly, the competition between the two responses may require valuable cognitive resources which may otherwise be devoted to plan execution itself (Webb, 2006). Having a Plan B may thus impose cognitive load and hinder the execution of (one of) the plan(s), in contrast to the swift and automatic enactment of the planned response when only one plan has been made.

This research aimed to (a) test whether making a Plan B yields less beneficial effects on goal pursuit compared to a 'conventional' single implementation intention, and (b) investigate its underlying mechanism(s). Two important gaps in the literature are thereby addressed. First, no research to date has examined the effects of multiple coping responses for one and the same situation on goal pursuit. Prior research examined the effects of multiple unique plans, each specifying a different situation and a different response. Second, it is unknown why making a Plan B may hinder goal pursuit. Previous studies show diverging results for the mechanism underlying multiple plans' unfavourable effects during plan formation. This research is the first to examine not only processes during plan formation, but also during plan enactment.

Two studies were conducted to test the hypothesis that making a Plan B is less beneficial to goal pursuit than making one implementation intention. In Study 1, the cognitive and motivational effects of making a Plan B during plan formation were examined. Specifically, it was examined whether a Plan B hinders the creation of strong mental associations between the situation and response(s) and/or decreases commitment to the plan(s). In Study 2, the behavioural effects of making a Plan B were examined, that is, whether a Plan B results in less-successful goal pursuit than one or no plan. In addition, the effects of making a Plan B during plan enactment were tested, i.e. whether a Plan B induces more cognitive load in the enactment phase than making one plan. In both studies, the focus was on eating behaviour as it constitutes a typical health goal that many people struggle with on a daily basis.

\section{Study 1}

In Study 1, we investigated whether specifying two responses for one situation ('making a Plan B') affects the cognitive association between the if-part and the then-part(s). A lexical decision task (LDT) was employed to measure the associative strength 
between the situation and the planned response(s). We expected that making a Plan B would lead to weaker associations between the situation and both planned responses, as evidenced by slower reaction times (RTs) to the planned response(s) after presentation of the situation, compared to formulating a single plan. In addition, we examined the alternative explanation that making a Plan B would decrease commitment to one or both of the plans.

\section{Method}

\section{Participants and procedure}

Participants were recruited from the university campus. Only females were included as they differ considerably from men in their (attitudes towards) eating behaviour (Wardle et al., 2004). In total, 171 females participated in the experiment. Upon arrival at the laboratory, all participants first provided informed consent, after which they were seated in front of a computer. Whereas half of the participants formulated one implementation intention (situation - Strategy A), the other half was asked to formulate an additional implementation intention specifying an alternative strategy for the same situation (situation - Strategy A and situation - Strategy B). Then, to measure the association strength between the situation and the strategy(ies), a primed LDT was employed to measure the accessibility of strategy A and B after a neutral cue prime (Neutral Prime) or the critical situation prime (Situation Prime). Next, control variables were measured to rule out alternative explanations (see below). Lastly, participants were probed for suspicion, debriefed, thanked and reimbursed for their participation.

After excluding eight participants because they were outliers on RTs to one of the two critical trials in the primed LDT ( $\mathrm{SD}>3 ; n=5)$, who indicated to be dyslectic $(n=1)$, or whose percentage correct answers in the LDT deviated substantially from the sample mean ( $\mathrm{SD}>3 ; n=2)$, the final sample consisted of 163 participants.

\section{Design}

The experiment had a 2 (Condition: Strategy A vs. Strategy A and B) $\times 2$ (Prime: Situation Prime vs. Neutral Prime) $\times 2$ (Order of Strategy Presentation in LDT: A first vs. B first; see below) between-subjects design.

\section{Materials \\ Pilot study}

As part of a larger data collection, pilot data were collected to assess the most frequently named situation in which female students consumed an unhealthy snack. Female participants $(N=289)$ with a mean age of 21.15 years $(\mathrm{SD}=2.87)$ and a mean BMI of $22.02(\mathrm{SD}=3.25)$ were asked to generate the situation in which they typically took an unhealthy snack. In addition, they indicated which strategy was most effective for them to resist the temptation in that situation. Results show that the most frequently named situation was 'boredom' $(N=59 ; 20.4 \%)$, and as research corroborates that boredom is an important trigger for eating unhealthily (Koball, Meers, Storfer-Isser, Dornoff, \& Musher-Eizenman, 2012), we chose 'boredom' as the critical situation (the 
if-part of the implementation intention). Regarding the most effective strategy for resisting temptation, results show that participants who named 'boredom' as the critical cue, the majority $(N=35 ; 59.3 \%)$ indicated that 'distraction' was the most effective strategy, followed by 'replacement' $(N=22 ; 37.3 \%)$. Consequently, 'distraction' was used as the first strategy, and 'replacement' as the second strategy for the then-part(s) of the implementation intention(s).

\section{Implementation intention formulation}

Participants first were given information concerning research demonstrating that 'boredom' is the most typical situation in which female students consume unhealthy snacks, and that 'distraction' was the most frequently used strategy to resist temptation when bored. All participants then formulated the intention 'I intend to reduce my unhealthy snack intake the coming week' by repeating it at least three times, after which they formulated implementation intentions. Specifically, they formulated 'If I am in the situation "boredom" and I feel like having a snack, then I will use the strategy distraction!'. To ensure sufficient encoding of the plan, participants were instructed to repeat the statement in their mind a few times, envision themselves acting out their plan and repeat the plan by typing it out. Next, they indicated how motivated they were to act out their plan on a 1 (not at all)-5 (very much) scale. Whereas half of the participants, the A condition, proceeded to the primed LDT (see below), the other half, the AB condition, received instructions to formulate an additional implementation intention for the same situation ('boredom'). They were given the following information: 'Sometimes you cannot execute your strategy in a situation. In such cases, it can be convenient to have another strategy at your disposal. We therefore would like to ask you to make a second plan'. Referring again to the results of the research indicating that 'replacement' is an additional widely used strategy, participants formulated the plan 'If I am in the situation 'boredom' and I feel like having a snack, then I will use the strategy replacement!'. For this second plan, sufficient encoding was also ensured, and motivation for the second plan was assessed similarly as for the first plan.

\section{Lexical decision task}

For the LDT, participants were instructed that they should press a left or a right key on a response box (Cedrus RB-530) to indicate as accurately and quickly as possible if a presented word was a word or non-word (corresponding keys for 'word' and 'non-word' were counterbalanced across participants). A practice task of eight trials was employed before proceeding to the actual LDT.

Each trial in the LDT started with a fixation cross $(1000 \mathrm{~ms})$, after which the prime was presented for $50 \mathrm{~ms}$. Next, a backward mask consisting of a string of x's was presented for $500 \mathrm{~ms}$, and then the target (word or non-word) appeared until participants responded by pressing a left or right key on the response box. After responding, a blank intertrial screen appeared for $2000 \mathrm{~ms}$.

The LDT constituted 80 trials in total, which consisted of 40 trials presented twice in fixed pre-randomised order in two sequential identical blocks. Primes and targets were matched on word length and frequency in the Dutch language and all targets were verbs to match the nature of the critical targets (the Dutch verbs 'afleiden' and 
'vervangen'). Targets included the two critical targets ('distraction' and 'replacement'), 18 neutral words (e.g. 'discover') and 20 pronounceable non-words (e.g. 'omstugen'). Primes included the critical situation 'boredom' (only in the Situation Prime Condition) or one of nine irrelevant words, resulting in each prime being presented four times. To ensure that RTs were not attributable to the order in which the critical targets were presented, half of the participants were presented with Strategy A before Strategy B and the other half vice versa.

\section{Control variables}

To rule out alternative explanations, we measured intention, habit strength and need for closure. The intention to reduce unhealthy snacking was measured twice by four items ('I intend/plan/want/expect to reduce my unhealthy snack intake in the near future'); once before the manipulation $(\alpha=.90)$ and once after the LDT $(\alpha=.92)$. The habit strength of consuming unhealthy snacks in the situation 'boredom' was measured by the 12-item Self-Report Habit Index (Verplanken \& Orbell, 2003). Each item started with "The consumption of unhealthy snacks in the situation "boredom" is something ...' followed by, e.g. ' $\ldots$ that is part of my routine' $(\alpha=.89)$. Need for closure was measured by a brief 15-item version of the Need for Closure Scale (Kruglanski, Webster, \& Klem, 1993; Roets \& Van Hiel, 2011). Example: 'I don't like situations that are uncertain' $(\alpha=.83)$. Need for closure reflects preference for predictability and being uncomfortable with ambiguity, and may therefore influence potential effects of making a Plan B.

\section{Strategy evaluations}

To measure participants' evaluations of the presented strategies to resist unhealthy snacks, three items assessed: (a) participants' use of the strategy, ranging from 1 (never) to 7 (always); (b) the extent to which they found the strategy effective; and (c) the extent to which they found the strategy effective in the situation 'boredom', ranging from 1 (totally disagree) to 7 (totally agree). Evaluations were assessed for the two strategies presented in the experiment, 'distraction' and 'replacement', as well as two filler strategies ('refuse' and 'ignore').

\section{Data analysis}

To check whether randomisation was successful, multiple ANOVAs were performed to test differences between the eight conditions (see 'Design') on intention, age, habit strength, need for closure and BMI. To analyse the LDT, incorrect responses were set to missing before analysis. As mean RTs were not normally distributed, RTs were natural log transformed. To facilitate interpretation, means and standard deviations are presented for the non-log transformed data. Separate ANCOVAs were performed for the RTs on Strategy A and on Strategy B, with the three between-subjects variables (Condition, Prime and Order of Strategy Presentation) as factors and BMI as a covariate. Only RTs for Block 1 were analysed, ${ }^{1}$ as repeated exposure to words facilitates subsequent lexical decisions and word naming performance to that word ('repetition priming effect'; Forster \& Davis, 1984). When a stimulus (pair) is presented, it is also primed, 
making later RTs to a repetitively presented stimulus (pair) less reliable than the first ones.

\section{Results}

\section{Descriptives and randomisation check}

Participants had a mean age of $20.58(\mathrm{SD}=2.80)$ and a mean BMI of 21.59 $(\mathrm{SD}=2.94)$. On average, participants had a moderate to high intention to reduce their unhealthy snacking $(M=4.73, \mathrm{SD}=1.44)$ and a moderate habit strength to eat unhealthy snacks in the situation 'boredom' $(M=3.89, \mathrm{SD}=1.21)$. The randomisation check showed no differences between conditions on the relevant variables (see 'Data analysis' (all $p \mathrm{~s}>.31)$ ), except for BMI, $F(7,155)=2.15, p=.04$. BMI was therefore included as covariate in all subsequent analyses.

\section{Main analyses}

RTs to Strategy A

The ANCOVA showed a significant interaction effect between Condition and Prime, $F(1,150)=4.54, p=.04, p \eta^{2}=.03$. Simple main effects demonstrated that within the Situation Prime condition, the A condition reacted faster to the target 'distraction' $(M=558.75, \mathrm{SD}=190.26)$ than the $\mathrm{AB}$ condition $(M=655.53, \mathrm{SD}=190.50), p=.07$. In contrast, within the Neutral Prime condition, RTs did not differ between the A condition $(M=610.42, \mathrm{SD}=189.52)$ and $\mathrm{AB}$ condition $(M=563.90, \mathrm{SD}=189.34), p=.26$. No other effects were significant, all $p \mathrm{~s}>.21$.

\section{RTs to Strategy B}

The same ANCOVA was performed for the RTs of the target 'replacement' (Strategy B) as for Strategy $\mathrm{A}^{1}$, which showed a significant interaction effect between Condition and Prime, $F(1,152)=6.25, p=.01, p \eta^{2}=.04$. Simple main effects showed that in the situation Prime condition, no differences emerged between the A condition $(M=575.60$, $\mathrm{SD}=156.99)$ and the $\mathrm{AB}$ condition $(M=613.00, \mathrm{SD}=156.85), p=.27$. In the Neutral Prime condition, the $\mathrm{AB}$ condition reacted faster $(M=526.63, \mathrm{SD}=155.57)$ to Strategy $\mathrm{B}$ than the A condition $(M=609.59, \mathrm{SD}=155.64), p=.02$. No other effects were significant, all $p \mathrm{~s}>.05 .^{2}$

\section{Motivational factor}

A repeated measures ANOVA showed that although overall participants' intention increased from before formulation of implementation intentions $(M=4.73, \mathrm{SD}=1.43)$ to after performing the LDT $(M=4.98, \mathrm{SD}=1.30), F(1,160)=4.09, p=.05$, this change in intention did not differ between the $\mathrm{A}$ and $\mathrm{AB}$ condition, $p=.93$ for the interaction effect. In addition, an ANOVA demonstrated that the A condition $(M=5.01$; $\mathrm{SD}=1.43)$ and the $\mathrm{AB}$ condition $(M=5.15 ; \mathrm{SD}=1.25)$ were equally motivated to execute their plan(s), $p=.50$. To examine whether RTs to the strategies were influenced by pre-existing attitudes towards the strategies, correlations were computed. In both the A 
and $\mathrm{AB}$ condition, none of the strategy evaluations was correlated with RTs (all $r \mathrm{~s}<.22$, all $p \mathrm{~s}>.12$ ).

\section{Discussion}

Study 1 demonstrated that making a Plan B decreases the association strength between the situation and Strategy A as compared to specifying only one implementation intention. Importantly, there was no stronger cognitive association between the critical situation and Strategy B for participants who made a Plan B compared to those formulating one plan. If that would have been the case - suggesting the execution of Strategy B rather than Strategy A - it would not necessarily be disadvantageous to specify an additional strategy: people could then still succeed in acting in line with their goal. The results, therefore, suggest that when making a Plan B, people are likely to refrain from executing either planned response.

These results are in line with prior research showing that formulating multiple implementation intentions leads to weakened cognitive associations between the situation (the if-part) and the planned strategy (the then-part; Verhoeven et al., 2013). Notably, making a Plan B did not influence intention or plan(s) commitment differently than formulating one implementation intention, which is in contrast to earlier research demonstrating that multiple implementation intentions compromise motivation for goal pursuit (Dalton \& Spiller, 2012). As such, the findings from Study 1 partly answer the question of why making a Plan B is detrimental to health goal striving: the formation of a strong cognitive association between situation and planned strategy is hindered, which is likely to result in a failure to execute the plan(s). Importantly, results show that motivational factors, such as intention or plan commitment, cannot explain why making a Plan B is harmful to health goal pursuit.

The finding that those who made a Plan B reacted faster to Strategy B (the strategy formulated in the second plan) than those who made one plan can be explained by the fact that making a Plan B entails familiarity with strategy B, and therefore, it requires less time to process the word. It should be noted that the Plan B condition reacted faster to Strategy $\mathrm{B}$ when it was preceded by a neutral prime rather than the critical situation, indicating that the link between the critical situation and Strategy B was not stronger for them.

Some limitations must be noted. This study demonstrated the negative effect of making a Plan B on cognitive processes rather than on actual behaviour. Moreover, it was only examined whether making a Plan B affects processes during plan formation. As elaborated on in the introduction, it is possible that making a Plan B also affects processes during plan enactment. Also, the strategies in the implementation intentions were experimenter given, which is at odds with the notion that self-determined personally relevant goals and plans are important to successful health goal pursuit. Lastly, there was no control condition, which prohibits conclusions about the effectiveness of plans in comparison with mere goal intentions.

Study 2 sought to overcome these limitations by the following adjustments. First, participants in Study 2 were able to choose their own coping response for their plan, rather than adopting an experimenter-given one. Second, the effects of making a Plan B were measured on actual behaviour. Third, a control group was included to examine the effects of making a Plan B compared to mere goal intentions as well as to a single implementation intention. 


\section{Study 2}

The first aim of Study 2 was to test the hypothesis that making a Plan B would be less beneficial for goal pursuit than making one or no plan, as assessed by actual behaviour within the critical situation. To demonstrate that making a Plan B has negative effects on goal pursuit regardless of the content and valence of the critical situation, a different situation was chosen (relaxation rather than boredom). As the assessment of behaviour was done in a controlled setting, the critical situation had to be standardised for all participants. Therefore, 'seeing chocolate' was chosen as the situation for which plans were made (in the planning conditions). It was not possible to recruit only participants who intended to reduce their unhealthy snack intake without jeopardising the credibility of the cover story and therefore the validity of the experimental set-up (see 'Procedure' below). Reducing chocolate intake was therefore chosen as an overarching goal that would apply to a large part of our sample. That is, chocolate is known to be a favourite snack among many women that is considered both attractive and conflicting with diet goals (Rozin, Levine, \& Stoess, 1991). The second aim was to examine whether making a Plan B would yield more cognitive load than making one plan during different stages of planning, which may explain the expected disadvantageous effects of making a Plan B. A Stroop task (Jostmann \& Koole, 2007) was therefore administered twice: Once after plan formation and once after plan enactment.

\section{Method}

\section{Participants and procedure}

Participants were recruited from university campus. The study, presented as an experiment about the influence of situational context on the ability to concentrate, consisted of two parts on two consecutive days. The design and cover story were chosen to minimise suspicion of the true purpose of the study. In total, 107 females participated in the first part of the experiment, but 12 failed to return for the second part. The final sample consisted of 95 participants.

\section{Day 1}

Upon arrival at the laboratory, participants were informed that they would perform a concentration task in two different rooms on two consecutive days. After providing informed consent, participants were then seated in front of a computer. Before they started the concentration task, the experimenter asked if they would be willing to fill out a short questionnaire for a colleague (scripted), which constituted the manipulation (see 'implementation intention formulation'). Afterwards, participants performed a modified Stroop task to measure cognitive load. It is important to note that the experimenter was blind to condition: All questionnaires were printed out before the experiment and put in a stack in random order.

\section{Day 2}

On the second day, participants were informed that we were also interested in the influence of relaxation on the ability to concentrate and therefore, they would first be 
allowed to relax for $10 \mathrm{~min}$ before performing the concentration task again (scripted). The participants were then taken to a room different from the previous day in which stood a relaxation chair, a coffee table with magazines and bowls with grapes and M\&Ms. The experimenter explained the purpose of the chair and the food (to help participants relax; scripted). The experimenter then left and kept track of the time with a stopwatch. To check whether participants adhered to instructions, participants were filmed via the built-in camera of the laptop, for which they consented both before and after the experiment. After $10 \mathrm{~min}$, the experimenter started the Stroop task on the laptop, and took the bowls with food and the magazines from the kitchen. The bowls were weighed to assess food intake. After the Stroop task, participants were asked about their strategy use during relaxation (see below), presented among filler items about their ability to concentrate and probed for suspicion. No participant guessed the true purpose of the study. Lastly, participants were debriefed, thanked and reimbursed for their participation.

\section{Design}

The experiment had three conditions (Condition: A vs. AB vs. Control group).

\section{Materials}

\section{Implementation intention formulation (Day 1)}

The questionnaire was ostensibly about New Year's resolutions to reduce unhealthy snack intake and the influence of plans on motivation. First, participants indicated how motivated they were to reduce their unhealthy snack intake, their average intake of various healthy and unhealthy snacks in a week (including chocolate and grapes, which were used for the assessment of food intake at Day 2), how much they liked these snacks and how motivated they were to reduce their intake of the unhealthy snacks. All items were measured on a seven-point scale ranging from 1 (not at all) to 7 (very much).

Then, participants were informed that we were interested in the extent to which making a plan about chocolate reduction would affect motivation. All participants formulated the intention to reduce chocolate intake in the near future. While the control condition merely repeated this intention by writing it down, the other two conditions (the $\mathrm{A}$ and $\mathrm{AB}$ condition) formulated one (two) implementation intention(s) to help them achieve their goal. Specifically, they formulated the plan 'If I see chocolate and I feel like having a snack, then I will use the strategy [self-chosen strategy]'. Participants chose their strategy from a list of four strategies: putting it out of sight, replacement, distraction or exerting willpower. Whereas the A condition proceeded to the final questions about motivation, the $\mathrm{AB}$ condition formulated an additional implementation intention for the same critical situation ('seeing chocolate'). Instructions for the $A B$ condition were the same as in Study 1, except that participants formulated 'If I see chocolate and I feel like having a snack, then I will use the strategy [another self-chosen strategy]' (also chosen from the listed strategies above). Sufficient encoding of the plan(s) was ensured by repeating and envisioning acting out the plan (see Study 1). 
Lastly, in addition to age, weight and height, all participants were asked to indicate on seven-point scales their (a) motivation to reduce their chocolate intake, (b) self-efficacy towards chocolate reduction, (c) intention to reduce their chocolate intake, (d) motivation to execute their plan(s). These questions were asked to make the cover story believable (i.e. the influence of plans on motivation), and they were included in the randomisation check.

\section{Modified Stroop task (Day 1 and 2)}

A simplified Stroop-colour-naming task was used to assess cognitive load (Jostmann \& Koole, 2007). Participants were presented with strings of coloured letters and were asked to respond to the font colour of the letters and ignore the meaning of the word. Letter strings represented a colour word (blue or red) or a series of Xs $(X X X X)$, which were displayed in red or blue font colour. If the words were presented in blue font colour, participants were asked to press the 6 key on the numeric pad of the keyboard; if the words were presented in red font colour, the $A$ key had to be pressed. Participants were instructed to keep their index fingers on the keys throughout the task, and to respond as fast and accurately as possible. A practice task of eight trials was presented before the actual task (only on Day 1).

The task consisted of 60 trials presented in random order: 20 congruent trials (red in red font colour or blue in blue font colour), 20 neutral trials ( $X X X X$ in red or blue font colour) and 20 incongruent trials (red in blue font colour or blue in red font colour). Each trial started with a fixation asterisk for $1000 \mathrm{~ms}$, followed by the presentation of a coloured letter string until response, followed upon response by a blank screen for $2000 \mathrm{~ms}$. The stimuli were presented in the centre of the screen of a desktop computer (Day 1) or a laptop (Day 2).

\section{Measured food intake (Day 2)}

Participants were presented with two bowls of food during relaxation: one with grapes and one with M\&Ms. Unbeknownst to participants, the bowls were weighed by the experimenter before and after the relaxation task to assess consumption of chocolate (M\&Ms) and the healthy alternative (grapes).

\section{Self-reported strategy use (Day 2)}

For each of the four strategies as presented to the $A$ and $A B$ condition the previous day (putting it out of sight, replacement, distraction and willpower), participants indicated the extent to which they used each of them during the relaxation task on seven-point scales.

\section{Control variables}

To ensure that potential food intake was not due to experimenter demand, participants were asked to what extent they felt free to take the available food on a seven-point scale, embedded among a similar item about reading the magazines. Also, to rule out alternative explanations, participants were asked whether they exerted effort to resist the 
M\&Ms (self-reported effort), to what extent they felt like having a snack (craving) and to what extent they found the M\&Ms tempting during relaxation (temptation) on seven-point scales.

\section{Data analysis}

As a randomisation check, multiple ANOVAs were performed to test differences between the three conditions on baseline motivation to reduce unhealthy snack intake, baseline intake of chocolate and grapes, liking of chocolate and grapes, motivation to reduce chocolate intake, evaluation of grapes as good alternative for unhealthy snacks, age and BMI. In addition, multiple ANOVAs were performed to examine whether the manipulation yielded unintended effects, as would be manifested in differences between conditions in motivation to reduce chocolate intake, self-efficacy towards chocolate reduction, intention to reduce chocolate intake and motivation to execute their plan(s).

To analyse differences between conditions in the amount of food intake for participants who ate unhealthy food, change scores were computed by subtracting the weight after relaxation from the weight before. Kilocalories were computed by multiplying grams with kcal per gram. In addition, scores were natural log-transformed as data were not normally distributed; non-transformed means and standard deviations are reported. To analyse alternative explanations for potential effects, multiple ANOVAs were performed on liking of chocolate, motivation, self-efficacy and intention to reduce chocolate intake, plan(s) motivation, the extent to which participants felt free to take the food, how tempting they found the M\&Ms, their craving and their exerted effort to resist the M\&Ms.

For the analyses of cognitive load, overall Stroop interference (faster colour-naming of neutral than incongruent stimuli) and facilitation (faster colour-naming of congruent than neutral stimuli) were analysed conducting several paired $t$-tests. Stroop Interference Scores (SIS) were computed by subtracting average responses on congruent trials from average responses on incongruent trials (Ståhl, van Laar, \& Ellemers, 2012). Higher scores on this measure indicate more Stroop interference and thus more cognitive load. Paired samples t-tests were conducted to analyse differences between Day 1 and Day 2 in SIS. To analyse differences between conditions in cognitive load, an ANOVA was performed for RTs on Day 1 and an ANCOVA for RTs on Day 2, with RTs on Day 1 as covariate.

\section{Results}

\section{Descriptives and randomisation check}

Participants had a mean age of $20.56(\mathrm{SD}=2.23)$ and a mean BMI of 21.39 $(\mathrm{SD}=2.49)$. On average, participants had a moderate to high motivation to reduce unhealthy snack intake $(M=4.53, \mathrm{SD}=1.30)$, scored high on liking of chocolate $(M=5.89, \mathrm{SD}=1.43)$ and moderate to high on liking of grapes $(M=5.39, \mathrm{SD}=1.42)$. The randomisation check showed that conditions did not differ on the relevant variables at baseline (all $p s>.13$ ), and there were no differences between conditions in variables that could have been unintended effects of the manipulation or that may have functioned as alternative explanations for potential results (all $p \mathrm{~s}>.19$; see 'Data analysis' for an overview of all variables that were analyzed). 


\section{Analyses of behaviour}

\section{Food intake percentages}

The non-forced eating setting allowed us to analyse the occurrence of eating per se (yes/no), and the amount consumed for those who ate (Taut, Brenner, \& Baban, 2012). A chi-square test showed that the number of participants that ate any M\&Ms did not differ by condition, $\chi^{2}(2)=.21, p=.90$, as well as the number of participants that ate any grapes, $\chi^{2}(2)=.91, p=.64$ (see Table 1$)$.

\section{Unhealthy food intake}

An ANOVA showed a significant main effect of condition, $F(2,61)=3.29, p=.04$, $p \eta^{2}=.10$. Simple main effects showed that whereas participants in the A condition and the Control condition ate an equal amount of calories in M\&Ms, $p=.76$, participants in the $\mathrm{AB}$ condition ate significantly more than both the A condition, $p=.02$, and the Control condition, $p=.05$ (see Table 1).

\section{Healthy food intake}

An ANOVA showed no differences between conditions in healthy food intake, $F(2,66)$ $=.42, p=.65$.

\section{Analyses of cognitive load \\ Stroop interference on Day 1 and Day 2}

Several paired $t$-tests showed that although there was interference on both days, facilitation was only present on Day 1 (for information on means and SDs on neutral, congruent and incongruent trials on both days, please contact the first author). In addition, it was revealed that on Day 1, there was more Stroop interference $(M=44.84$, $\mathrm{SD}=62.21)$ than on Day $2(M=18.79, \mathrm{SD}=44.58), t(65)=3.60, p<.01$, indicating a learning effect. As SIS on Day 1 and Day 2 were significantly correlated $\left(r=.34^{* *}\right)$, we included SIS of Day 1 as a covariate for the analyses on Day 2.

\section{Differences between conditions in cognitive load}

Before analysis, incorrect responses $(2.3 \%$ on both days), responses that were extremely fast $(<300 \mathrm{~ms} ; 2.9 \%$ on Day 1 and $1.2 \%$ on Day 2 ; see Jostmann \& Koole, 2007) were

Table 1. Differences between conditions on outcome measures of Study 2.

\begin{tabular}{|c|c|c|c|c|c|c|c|}
\hline & \multirow[b]{2}{*}{$N$} & \multicolumn{2}{|c|}{ A } & \multicolumn{2}{|c|}{$\mathrm{AB}$} & \multicolumn{2}{|c|}{ Control } \\
\hline & & $M$ & SD & $M$ & SD & $M$ & SD \\
\hline Participants who ate healthy foods (\%) & 95 & 76.5 & & 66.7 & & 67.7 & \\
\hline Participants who ate unhealthy foods (\%) & 95 & 64.7 & & 70.0 & & 67.7 & \\
\hline Healthy food intake $(\mathrm{g})$ & 69 & 41.12 & 35.84 & 34.80 & 26.49 & 35.88 & 45.56 \\
\hline Unhealthy food intake (kcal) & 64 & $59.81^{\mathrm{a}}$ & 87.78 & $97.73^{\mathrm{b}}$ & 98.19 & $60.76^{\mathrm{a}}$ & 73.04 \\
\hline Unhealthy food intake $\left(\mathrm{g} / \mathrm{n}^{\mathrm{o}}\right.$ of $\left.\mathrm{M} \& \mathrm{Ms}\right)$ & 64 & $19.14^{\mathrm{a}}$ & 19.59 & $18.57^{\mathrm{b}}$ & 15.04 & $28.90^{\mathrm{a}}$ & 18.33 \\
\hline Stroop interference & 72 & $1.27^{\mathrm{b}}$ & 38.80 & $22.15^{\mathrm{a}}$ & 39.87 & $33.91^{\mathrm{a}}$ & 49.73 \\
\hline
\end{tabular}

Note: Means with different superscripts differ at $p \leq .05$. 
set to missing. Participants who responded extremely slow on the means of neutral, congruent or incongruent trials $(>2.5 \mathrm{SD})$ were excluded from analysis $\left(N_{\text {Day } 1}=1\right.$, $N_{\text {Day } 2}=4 ; 5.4 \%$ of participants). In addition, due to a technical error, ${ }^{3}$ data from 23 participants were unusable and thus excluded from analysis. Consequently, results for Stroop interference are reported for 72 participants. (RTs) were log-transformed; descriptives are reported for non-transformed RTs (see Table 1). An ANOVA showed that condition had no significant effect on Stroop interference on Day $1, F(2,63)=1.66$, $p=.20$. On Day 2, in contrast, an ANCOVA showed that condition had a significant effect on Stroop interference, $F(2,62)=3.16, p=.05, p \eta^{2}=.09$. As Table 1 shows, whereas the A condition showed almost no Stroop interference, the $\mathrm{AB}$ condition and Control condition did. Pairwise comparisons showed that whereas the A condition experienced less cognitive load than both the $\mathrm{AB}$ and Control conditions, $p=.05$ and $p=.03$, the $\mathrm{AB}$ and Control condition did not differ, $p=.81$.

\section{Discussion}

The first aim of Study 2 was to examine the effects of making a Plan B on actual behaviour. Findings show that people who made a Plan B to resist chocolate ate more chocolate than those who made one or no plan. Unexpectedly, the percentage of participants that refrained from eating any of the unhealthy food was equal among conditions and no differences emerged in unhealthy food intake between participants who formulated one plan and those in the control condition. This implies that making one, two or no plan(s) may to some extent be equally effective in successful goal pursuit, even considering that people who made a Plan B ate more unhealthy food than the other two conditions. It should be noted that dealing with the critical situation was more cognitively taxing for participants who made two or no plans, as evidenced by greater Stroop interference, compared to those who made one plan. This indicates that it may have taken more effort to resist the temptation of chocolate for those who made a Plan B or no plan at all. As it has been shown that self-regulatory failure often occurs after initial acts of self-control (Baumeister, Gailliot, DeWall, \& Oaten, 2006), it is possible that participants who made one plan would have been more successful in self-regulation if a subsequent self-regulatory task had been performed.

The second aim was to investigate whether cognitive load during plan enactment could provide an explanation for the detrimental effects of making a Plan B. Results demonstrate that having made a Plan B led to more cognitive load than having made one plan during the plan enactment stage. Notably, there were no differences in cognitive load immediately after plan formation, which rules out the alternative explanation that forming multiple plans taxes self-regulatory resources during plan formation (Hagger \& Luszczynska, 2014). Similar to Study 1, motivational factors did not differ between conditions. This study therefore shows that making a Plan B may not affect motivation or cognitive resources during plan formation, but rather hinders acting upon the plans by imposing cognitive load during plan enactment.

\section{General discussion}

This research aimed to investigate the effect of specifying two coping strategies for one critical situation ('making a Plan B') on successful goal pursuit, and its underlying 
mechanisms. Both studies reveal that, as hypothesised, making a Plan B is less beneficial to health goal pursuit than formulating one implementation intention. In addition, results demonstrate that it interferes with crucial cognitive processes during both plan formation (creation of strong associative links; Study 1) and plan enactment (the absence of cognitive load; Study 2). Together, these results suggest that, despite its intuitive appeal, planned flexibility may come at the cost of successful goal pursuit.

The first aim of this research was to investigate whether increased planned flexibility would facilitate successful goal striving in the context of self-regulation. The hypothesis that making a Plan B is less beneficial to goal pursuit than formulating one implementation intention received consistent support in both studies. Making a Plan B did not only undermine the creation of strong mental situation-response links necessary for successful goal pursuit (Gollwitzer, 1999), but also negatively affected actual successful goal pursuit. These findings are consistent with studies showing that multiple plans are not necessarily more beneficial to successful goal striving than one plan (Armitage \& Arden, 2012) or even detrimental (Dalton \& Spiller, 2012; Verhoeven et al., 2013). As such, this research builds upon the discussion about ways to address the need for more flexibility in plans (Hagger \& Luszczynska, 2014; Webb, 2006). Plans can be easily hindered by environmental factors during plan enactment (e.g. not having the opportunity to execute a planned strategy) which necessitates flexibility, as was also demonstrated by recent research (Bayuk et al., 2010; Masicampo \& Baumeister, 2012). The present studies complements this research by showing that formulating several strategies (making a Plan B) may not be the solution to address this need for flexibility. Nonetheless, it would be premature to conclude that making a Plan B never works. There may be circumstances or ways in which planned flexibility does facilitate goal pursuit, for example, when people include two strategies within the same plan, e.g. 'If I see chocolate, then I will use strategy A or B' (cf. Wieber et al., 2009). Also, it is possible that multiple plans may not be necessarily detrimental to health goal attainment compared to one plan if people are given the choice to form more than one plan rather than being 'forced' to do so (cf. Armitage \& Arden, 2012). Future research should address these suggestions.

The second aim was to examine the underlying mechanisms of the hypothesised negative effects of making a Plan B. The literature advanced three possible mechanisms: making a Plan B may (1) hinder the creation of strong cue-action links during plan formation; (2) reduce motivation to attain the goal or execute the plan(s) after plan formation; and (3) impose cognitive load during plan enactment. The present studies demonstrate that people who made a Plan B failed to establish strong cue-action links, whereas their motivation to execute the plans remained intact compared with people who made one plan. This result is in line with prior research showing that it is cognitive rather than motivational processes that are responsible for the ineffectiveness of multiple plans (Verhoeven et al., 2013). However, Dalton and Spiller (2012) found that making multiple plans for multiple goals highlights the difficulty of attaining them all, thereby undermining goal commitment. Although this research seems to indicate the involvement of motivational processes, the present studies show that making multiple plans does not negatively affect plan and goal commitment and goal intentions. As ample research has shown that these processes can also drive goal pursuit on an implicit level (e.g. Aarts, Gollwitzer, \& Hassin, 2004), it is an important avenue for future research to examine whether multiple plans influence motivation implicitly. 
The third mechanism - cognitive load during the plan enactment stage - was also shown as a plausible explanation for the detrimental effects of making a Plan B. The absence of cognitive resources in planning processes during plan formation has been put forward to explain why multiple plans may not work (Hagger \& Luszczynska, 2014), but the present research ruled out this explanation. Instead, having a Plan B taxed cognitive resources during plan enactment, which raises the question as to why a Plan B results in depleted resources. It is possible that having a Plan B invokes the need to scrutinise the suitability of the planned coping response in situ, leaving ample opportunity for interference with the execution of any planned response (Gollwitzer, 1999). As this is yet unknown, an important avenue for future research is to replicate and further examine the role of cognitive resources during plan enactment. Also, as this research examined mechanisms in separate studies rather than in conjunction, it is worthwhile for future studies to examine the relative contribution of the aforementioned mechanisms to the negative effects of making a Plan B.

This research makes several important contributions to the literature. First, this is the first study to show that making a Plan B is disadvantageous to goal pursuit, which addresses important questions about flexibility in planning that have not been investigated to date (Hagger \& Luszcsynska, 2014). Second, this research advances the novel notion that the ineffectiveness of making multiple plans may not only be explained by its interference with cognitive processes during the plan formation stage, as has been shown before (e.g. Verhoeven et al., 2013), but also during the plan enactment stage. This research thus sheds light on underlying mechanisms of implementation intentions during different stages of planning that have seldom been examined to date. The current studies' insights can help explain why some planning interventions are less successful than others, and importantly, can be used for the development of new planning interventions aimed at health behaviour change.

Several limitations have to be noted. First, the effects of making a Plan B were investigated in a - albeit as natural as possible - lab context that cannot directly be extrapolated to goal pursuit in real life settings. Although the controlled settings may have provided the most stringent test, future research should attempt to replicate the findings in a more ecologically valid setting. Second, cognitive resources were measured after participants were able to eat food, which may have influenced cognitive load and precludes a causal conclusion about cognitive load during plan enactment. This is an important issue for further studies to address.

To conclude, contrary to the intuitive belief that planned flexibility in goal-directed responses to an uncertain and unpredictable goal-threatening situation is a blessing, it may in fact sometimes be a curse. Rather than promoting efficient, effortless and successful goal attainment - as is assumed to occur by planning - planned flexibility increases the likelihood that self-regulation failure will occur. When it comes to planning, then, it may actually be better to be sorry than safe.

\section{Funding}

This research was supported by a grant from ZonMw, the Netherlands organization for health research and development [grant number 120610009]. 


\section{Notes}

1. For the mean RTs on Strategy A of both blocks, the ANCOVA showed a marginally significant interaction effect between Condition and Prime, $F(1,150)=2.76, p=.10$. For the mean RTs on Strategy B for both blocks, the ANCOVA showed a significant interaction effect between Condition and Prime, $F(1,150)=4.35, p=.04, p \eta^{2}=.03$.

2. A marginally significant interaction between Condition and Order of Strategy Presentation emerged, $F(1,152)=3.67, p=.06$. When 'distraction' (Strategy A) was presented first in the LDT, there were no differences between the A condition $(M=575.13, \mathrm{SD}=157.28)$ and the $\mathrm{AB}$ condition $(M=596.21, \mathrm{SD}=157.57)$ in RTs to 'replacement' (Strategy B), $p=.49$. However, when 'replacement' (Strategy B) was presented first in the LDT, the AB condition reacted faster $(M=543.42, \mathrm{SD}=155.70)$ to 'replacement' (Strategy B) than the A condition $(M=610.06, \mathrm{SD}=155.51), p=.05$. Importantly, these effects were independent of whether participants were presented with the Situation Prime or a Neutral Prime before the strategy, and therefore, are not relevant to the critical situation-response associations that were the main interest of this research. All other $p \mathrm{~s}>.14$.

3. Two different technical errors occurred. For nine participants, the display settings of the laptop were incompatible with the default display settings in $E$-prime, causing both the fixation cross and the letter strings to be displayed at the left rather than the centre of the screen. For an additional 14 participants, rather than displaying the fixation cross and letter string in the centre, the letter string was presented at the far right end of the screen, while the fixation cross was at the centre.

\section{References}

Aarts, H., Gollwitzer, P. M., \& Hassin, R. R. (2004). Goal contagion: Perceiving is for pursuing. Journal of Personality and Social Psychology, 87, 27-37.

Adriaanse, M. A., Vinkers, C. D. W., De Ridder, D. T. D., Hox, J. J., \& De Wit, J. B. F. (2011). Do implementation intentions help to eat a healthy diet? A systematic review and meta-analysis of the empirical evidence. Appetite, 56, 183-193.

Armitage, C. J., \& Arden, M. A. (2012). A volitional help sheet to reduce alcohol consumption in the general population: A field experiment. Prevention Science, 13, 635-643.

Baumeister, R. F., Gailliot, M., DeWall, C. N., \& Oaten, M. (2006). Self-regulation and personality: How interventions increase regulatory success, and how depletion moderates the effects of traits on behavior. Journal of Personality, 74, 1773-1802.

Bayer, U. C., Achtziger, A., Gollwitzer, P. M., \& Moskowitz, G. B. (2009). Responding to subliminal cues: Do if-then plans facilitate action preparation and initiation without conscious intent? Social Cognition, 27, 183-201.

Bayuk, J. B., Janiszewski, C., \& Leboeuf, R. A. (2010). Letting good opportunities pass us by: Examining the role of mind-set during goal pursuit. Journal of Consumer Research, 37, 570-583.

Brandstädter, J., \& Rothermund, K. (2004). The life-course dynamics of goal pursuit and goal adjustment: A two-process framework. Developmental Review, 22, 117-150.

Dalton, A. N., \& Spiller, S. A. (2012). Too much of a good thing: The benefits of implementation intentions depend on the number of goals. Journal of Consumer Research, 39, 600-614.

Forster, K. I., \& Davis, C. (1984). Repetition priming and frequency attenuation. Journal of Experimental Psychology: Learning, Memory and Cognition, 10, 680-698.

Gollwitzer, P. M. (1999). Implementation intentions: Strong effects of simple plans. American Psychologist, 54, 493-503.

Gollwitzer, P. M. (2006). Open questions in implementation intention research. Social Psychology Review, 8, 14-18.

Gollwitzer, P. M., Wieber, F., Myers, A. L., \& McCrea. (2010). How to maximize implementation intention effects. In C. R. Agnew, D. E. Carlston, W. G. Graziano, \& J. R. Kelly (Eds.), 
Then a miracle occurs: Focusing on behavior in social psychological theory and research (pp. 137-161). New York, NY: Oxford Press.

Hagger, M. S., \& Luszczynska, A. (2014). Implementation intention and action planning interventions in health contexts: State of the research and proposals for the way forward. Applied Psychology: Health and Well-being, 6, 1-47.

Jostmann, N. B., \& Koole, S. L. (2007). On the regulation of cognitive control: Action orientation moderates the impact of high demands in Stroop interferences tasks. Journal of Experimental Social Psychology, 136, 593-609.

Koball, A. M., Meers, M. R., Storfer-Isser, A., Domoff, S. E., \& Musher-Eizenman, D. R. (2012). Eating when bored: Revision of the emotional eating scale with a focus on boredom. Health Psychology, 31, 521-524.

Kruglanski, A. W., Webster, D. M., \& Klem, A. (1993). Motivated resistance and openness to persuasion in the presence or absence of prior information. Journal of Personality and Social Psychology, 65, 861-876.

Masicampo, E. J., \& Baumeister, R. F. (2012). Committed but closed-minded: When making a specific plan for a goal hinders success. Social Cognition, 30, 37-55.

Roets, A., \& van Hiel, A. (2011). Item selection and validation of a brief, 15 -item version of the need for closure scale. Personality and Individual Differences, 50, 90-94.

Rozin, P., Levine, E., \& Stoess, C. (1991). Chocolate craving and liking. Appetite, 17, 199-212.

Shah, J. Y., Friedman, R., \& Kruglanski, A. W. (2002). Forgetting all else: On the antecedents and consequences of goal shielding. Journal of Personality and Social Psychology, 83, $1261-1280$.

Ståhl, T., van Laar, C., \& Ellemers, N. (2012). The role of prevention focus under stereotype threat: Initial cognitive mobilization is followed by depletion. Journal of Personality and Social Psychology, 102, 1239-1251.

Taut, D., Brenner, B., \& Baban, A. (2012). Reappraise the situation but express your emotions: Impact of emotion regulation strategies on ad libitum food intake. Frontiers in Psychology, 3, $1-7$.

Verhoeven, A. A. C., Adriaanse, M. A., De Ridder, D. T. D., de Vet, E., \& Fennis, B. (2013). Less is more: The effect of multiple implementation intentions targeting unhealthy snacking habits. European Journal of Social Psychology, 43, 344-354.

Verplanken, B., \& Orbell, S. (2003). Reflections on past behavior: A self-report index of habit strength. Journal of Applied Social Psychology, 33, 1313-1330.

Wardle, J., Haase, A. M., Steptoe, A., Nillapun, M., Jonwutiwes, K., \& Bellisie, F. (2004). Gender differences in food choice: The contribution of health beliefs and dieting. Annals of Behavioral Medicine, 27, 107-116.

Webb, T. L. (2006). Getting things done: Self-regulatory processes in goal pursuit. Social Psychological Review, 8, 2-13.

Wrosch, C., Scheier, M. F., Miller, G. E., Schulz, R., \& Carver, C. S. (2003). Adaptive self-regulation of unattainable goals: Goal disengagement, goal reengagement, and subjective well-being. Personality and Social Psychology Bulletin, 29, 1494-1508. 